\title{
The role of released ATP in killing Candida albicans and other extracellular microbial pathogens by cationic peptides
}

\author{
Slavena Vylkova $\cdot$ Jianing N. Sun $\cdot$ Mira Edgerton
}

Received: 24 April 2006 / Accepted: 30 May 2006 / Published online: 8 February 2007

(C) Springer Science + Business Media B.V. 2007

\begin{abstract}
A unifying theme common to the action of many cationic peptides that display lethal activities against microbial pathogens is their specific action at microbial membranes that results in selective loss of ions and small nucleotides - chiefly ATP. One model cationic peptide that induces non-lytic release of ATP from the fungal pathogen Candida albicans is salivary histatin 5 (Hst 5). The major characteristic of Hst 5-induced ATP release is that it occurs rapidly while cells are still metabolically active and have polarized membranes, thus precluding cell lysis as the means of release of ATP. Other cationic peptides that induce selective release of ATP from target microbes are lactoferricin, human neutrophil defensins, bactenecin, and cathelicidin peptides. The role of released extracellular ATP induced by cationic peptides is not known, but localized increases in extracellular ATP concentration may serve to potentiate cell killing, facilitate further peptide uptake, or function as an additional signal to activate the host innate immune system at the site of infection.
\end{abstract}

S. Vylkova $\cdot$ J. N. Sun $\cdot$ M. Edgerton

Department of Oral Biology, School of Dental Medicine,

State University of New York at Buffalo,

Buffalo, NY 14214, USA

\section{Edgerton}

Restorative Dentistry, School of Dental Medicine,

State University of New York at Buffalo,

Buffalo, NY 14214, USA

M. Edgerton ( $\square)$

State University of New York at Buffalo,

310 Foster Hall, SUNY at Buffalo Main Street Campus,

3435 Main Street,

Buffalo, NY 14214, USA

e-mail: edgerto@buffalo.edu
Key words ATP release $\cdot$ Candida albicans $\cdot$ cationic antimicrobial peptides $\cdot$ defensins $\cdot$ histatin

\section{Introduction}

Microbes come in contact with naturally occurring cationic proteins from many sources [1]. Fluids and mucosal surfaces in the oral cavity, lacrimal fluids, and gut are all rich in peptides that limit the colonization and overgrowth of indigenous bacteria and fungi. Their protective functions become evident upon loss of these peptides, which often results in dramatic bacterial and fungal overgrowth. Thus, conditions that reduce salivary flow in the oral cavity predispose host tissues to candidiasis and increased bacteria colonization [2].

Understanding the mechanism by which these cation peptides limit microbial growth is important not only because of the crucial role that these innate peptides play in human health, but also because of the possibility of therapeutic use of naturally occurring peptides [3, 4]. Therefore, the mechanism by which host-derived peptides distinguish bacterial and fungal targets from self is a fundamental question. In addition, the discrimination mechanism by which these peptides exert lethal effects on microbes, but not on host tissues, is central to their potential use as alternative or complementary drug therapies. One unifying theme that is common to many peptides that display lethal activities against microbial pathogens is their specific action at microbial membranes that results in selective loss of ions and small nucleotides - chiefly ATP. The commonality of this mode of action and the significance of selective ATP release are themes discussed in this review. 


\section{What are shared mechanisms of action among cationic peptides causing ATP release?}

Cytoplasmic membrane dysfunction through pore formation appears to be a common mechanism of action of many antimicrobial peptides active against bacteria [5-7]. Peptides initially bind with the target microbe membrane via electrostatic interactions with the negatively charged surface or by binding with lipopolysaccharides (LPS) in the bacterial outer membrane. After binding of peptide monomers has occurred, the peptide is inserted into membranes, followed by self-association and formation of localized aggregates, and culminating in formation of membrane pores. Peptide-induced pores allow release of ATP, ions, and other molecules, membrane depolarization, osmotic dysregulation, and ultimately cell death. Examples of such mechanisms of killing between peptides and their membrane targets include magainin 2 in gram-negative bacteria [8], thrombin-induced platelet microbicidal protein [9], and human defensins and lactoferrin $[10,11]$.

An alternative mechanism to nonspecific pore formation as a route to release ATP from cells is peptide association with specific proteins within target cell membranes that results in loss of function of the membrane protein. Such interactions confer specificity of the peptide for a vulnerable cell. Many naturally occurring toxins exert their effects through inhibition of sodium or potassium ion channel functions $[12,13]$. These small peptides (22-60 amino acid residues) are the venoms produced by diverse animal species such as scorpions, snakes, spiders, and sea anemones. Such toxins blockade ion channels, either by direct occlusion of the pore or by causing allosteric modification to the channel. Other toxins may indirectly affect channel function by alteration of other channel-interacting molecules such as enzymes [14]. In either case, the result is loss of the cellular ion gradient, which in turn causes membrane depolarization or inactivation of potassium-dependent enzymes and pathways. In order to recover and stabilize the membrane potential, the cell compensates by activating other membrane proteins, such as ATP hydrolyzing pumps or channel-mediated transport of $\mathrm{Na}^{+}$and $\mathrm{Cl}^{-}$, and thus requires utilization of cellular energy [15].

\section{Cationic peptides induce ATP release integral to microbicidal activity}

\section{Histatins}

In terms of mechanism of antimicrobial activity, perhaps one of the best studied cationic peptides causing non-lytic release of ATP is the salivary histatin 5 (Hst 5). Histatin 5 first binds to Candida albicans cell wall proteins [16], identified as Ssa1/2 proteins from the heat shock 70 family [17], which facilitate intracellular transport to other effector sites for its toxic activity. Hst 5 binding, translocation, and toxicity are closely related processes, and loss of either Ssa1 or Ssa2 proteins results in diminished Hst 5 intracellular transport and cell killing [17]. Similarly, internalization of histatin variant peptides dhvar4 and dhvar5 has been demonstrated $[18,19]$, but it is not clear whether they also require binding to ssa $1 / 2 \mathrm{p}$ on the fungal cell envelope for their activity. A specific binding site for Hst 5 has been identified on Porphyromonas gingivalis as well, showing that the antimicrobial activity of Hst 5 may be mediated by binding to specific receptor(s) on the cell surface [20].

One of the most prominent features of salivary histatins and variant peptides killing of $C$. albicans is the rapid efflux of cellular ATP and other small nucleotides such as $\mathrm{NAD}^{+}$, AMP, ADP, and ions, as well as concurrent intracellular uptake of the DNA-binding dye propidium iodide [19, 21, 22]. Histatin-induced ATP release does not require extracellular binding, since these effects can be initiated solely from intracellular sites [23]. ATP efflux and depletion of intracellular ATP in C. albicans cells following Hst 5 treatment has been shown to be concentration and time dependent, with maximal depletion of about $85 \%$ of intracellular ATP occurring within $30 \mathrm{~min}$ of exposure to physiological concentrations of Hst 5 [21]. The major characteristic of Hst 5-induced ATP release was that it occurred while $C$. albicans cells were metabolically active and had polarized membranes, thus precluding cell lysis as a possible route by which ATP was released from the cells [21]. Interestingly, Hst 5 killing of anaerobic cultures was reduced compared to cells grown under air, although incubation of anaerobically grown cells with Hst 5 caused a marked increase in extracellular ATP (ATPe) and a drastic depletion of cellular ATP compared to the untreated cells [21]. Pretreatment of $C$. albicans cells with ion transport inhibitors, such as 4,4'-diisothiocyanlstilbene-2, 2'-disulfonic acid (DIDS), and niflumic acid all blocked ATP loss and killing in Hst 5-treated C. albicans, suggesting that Hst 5 activity is mediated by ion transporter(s) on yeast plasma membrane [24]. Indeed, deletion of TRK1, the major plasma membrane $\mathrm{K}^{+}$uptake system in C. albicans, nearly abolished Hst 5-initiated ATP release, cell killing, and cellular permeability for propidium iodide [25, 26, 27], indicating that Hst 5 ultimately results in alteration of Trk $1 \mathrm{p}$ function. The current model for Hst 5 activity, which may be similar to other cationic peptides, is shown in Fig. 1.

Whether the ATPe released from C. albicans in response to Hst 5 might have a further physiological role in cell death is not clear. Previously we suggested that ATP released from $C$. albicans by Hst 5 may auto-activate membrane receptors to cause cell death, although we have 


\section{Cell wall}

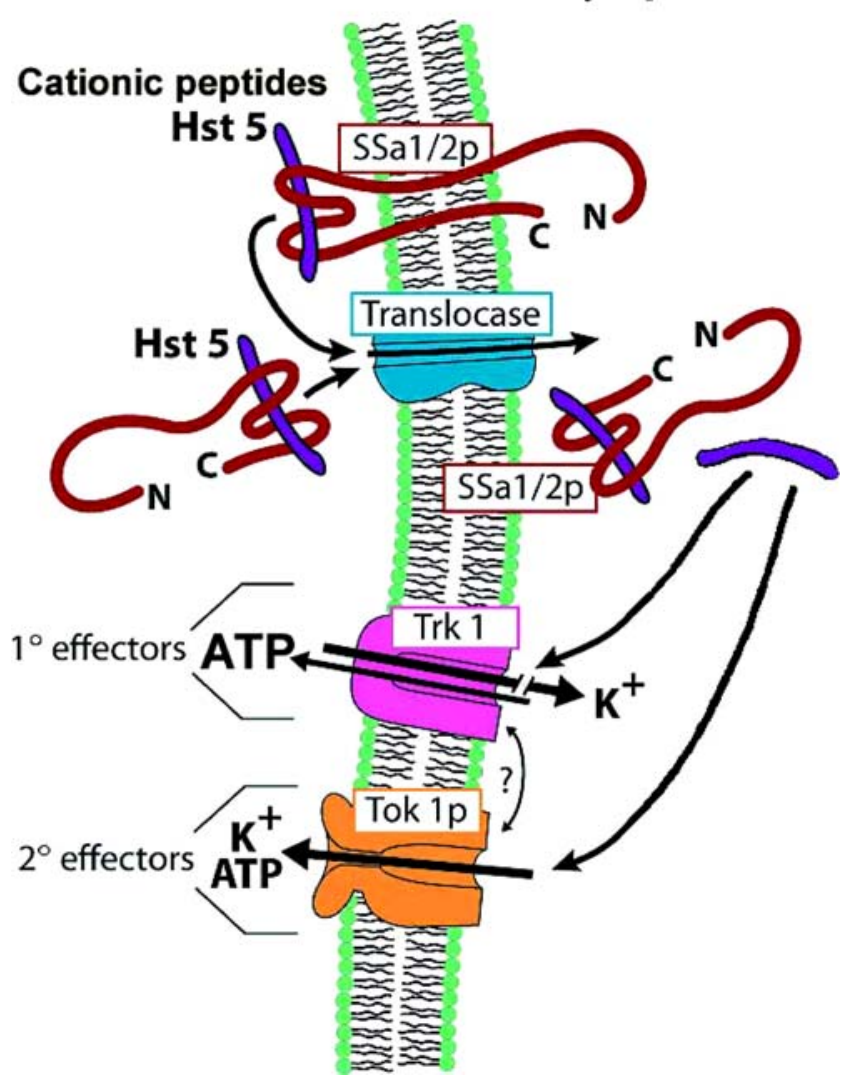

Fig. 1 Mechanism of action of histatin 5 with target Candida albicans cells

not been able to identify conventional purinergic receptors in C. albicans cells [21]. In higher eukaryotic cells, released purine nucleotides and nucleosides interact with cell surface receptors (purinoreceptors) to induce a range of biological effects including cytotoxicity [28]. In this regard, application of the peptide antibiotic polymyxin B to immune cells synergized plasma membrane permeabilization induced by ATP, so that cell killing was enhanced by the combined application of both ATP and polymyxin B [29]. For fungal cells, treatment of $C$. albicans cells for $3 \mathrm{~h}$ with high doses of $\mathrm{P}_{2} \mathrm{X}_{7}$-selective ATP analogues BzATP, ATP $\gamma \mathrm{S}$, and $\mathrm{ATP}^{4-}$ caused about a $60 \%$ loss of viability of aerobically grown cells, suggesting that ATPe itself may potentiate cell killing [21]. Pretreatment of $C$. albicans cells with the phosphatase apyrase (an ATP-consuming enzyme) greatly reduced Hst 5-induced cell death [21], further supporting the requirement for available ATPe in Hst 5 activity. In the case of Hst 5-treated cells, released ATP may act upon cells already severely depleted of ATP, so that cells may be more prone to ATP-mediated killing in a synergistic manner with Hst 5. Alternatively, Hst 5 entry into the cells may itself require ATP. In this respect, ATP might facilitate Hst 5 binding to $s s a 1 / 2 p$ on the cell envelope, since heat shock proteins function in a strongly ATP-dependent manner [30].
Whether Ssa proteins utilize intracellular and/or ATPe for Hst 5 binding and translocation has not been demonstrated, but since inhibition of mitochondrial ATP synthesis protected C. albicans from the fungicidal activity of histatin 5 and this protection appeared to be due to reduced cellular accumulation of the peptide [31], such a possibility should be considered.

Lactoferrin and derived peptides

Lactoferrin is an abundant $77-\mathrm{kDa}$ iron-binding glycoprotein found in saliva. The iron-binding domain of the lactoferrin molecule is located at the carboxy terminus, while the highly basic N-terminal region contains a 25 amino-acid microbicidal domain termed lactoferricin (LF). The N-terminus of LF, $\operatorname{LF}(1-11)$ consisting of only 11 amino acid residues, retains complete bactericidal and antifungal properties [32].

LF(1-11) appears to have similar features in its antifungal activity to histatin 5, in that it induces extracellular release of ATP and other nucleotides and ions [33], and subsequent loss of viability occurs in an energydependent manner [32]. These effects were not related to extensive membrane permeabilization [34] since LF did not cause release of macromolecular cytosolic constituents and was unable to disrupt the liposomal phospholipid bilayer in vitro [35]. In addition, LF was internalized prior to cell death [36], thus indicating that LF affects cytoplasmic targets rather than forming pores in plasma membrane of susceptible fungi.

Like Hst 5, LF(1-11) caused a time- and dosedependent increase in ATPe, which was completely blocked by pretreatment of cells with azide [32]. ATPe seems to play a role in the killing of Candida cells following treatment with peptides derived from the N-terminus of human lactoferrin, since preincubation with oATP, which irreversibly blocks the interaction between ATPe and extracellular receptors, resulted in significant protection from killing [37]. However, addition of ATP or ATP analogues (such as BzATP and ATP $\gamma \mathrm{S}$ ) was not sufficient to kill $C$. albicans, nor did ATP act synergistically with LF peptide to induce $C$. albicans cell death $[32,37]$.

Despite the striking similarities to the Hst 5 mechanism of antifungal action, lactoferricin killing of $C$. albicans has unique steps as well. In this respect, $C$. albicans spheroplast susceptibility to human LF was significantly reduced following treatment with proteinase $\mathrm{K}$ [33], thus suggesting the possibility that LF-induced killing is mediated by specific protein receptor(s) on the cell surface. However, our results showed that $C$. albicans susceptibility to $\operatorname{LF}(1-$ 11) was not altered in $S S A$ mutant strains [27], thus suggesting that the Hst 5 Candida cell wall binding protein ssa $1 / 2 p$ is not a binding site for LF. 
We have shown that TRK1-deficient strains are resistant to LF peptides compared to wild-type cells. As for Hst 5, TRK1 knockdown strains were protected from cell death and concurrent propidium iodide uptake induced by LF [27]. Although we did not quantify the effect of LF on ATPe release in TRK1-deficient cells, ATP release correlates with the percentage of PI-positive cells as previously reported [32]. Similarly to the effect of salivary Hst 5, LF treatment of $C$. albicans cells seems to involve transportermediated efflux of intracellular ATP and ions, which contributes to cell death [38]. Thus, LF may modulate the function of Candida cell membrane transporters, and possibly other proteins maintaining membrane integrity. In this context, the synthetic D-decapeptide BM2 (D- $\mathrm{NH}_{2}-$ RRRFWWFRRR-CONH$H_{2}$, which shares the first nine residues of $L F(1-11)$, acts as a specific inhibitor of the yeast plasma membrane ATP-driven proton pump Pmalp [39]. However, we found that LF(1-11)-induced killing of $P M A 1$ knockdown strains was undistinguishable from that of wild-type cells (unpublished data), thus indicating that LF(1-11) does not exert its effects by affecting Pmalp function.

\section{Bactenecins}

Bactenecins are proline-rich peptides processed from precursor cathelicidins, residing with other antimicrobial peptides in large granules within polymorphonuclear neutrophils. Bactenecins are active against gram-positive, gram-negative bacteria and fungi. Similar to antibacterial activity of LF, bactenecin and derived peptides have been shown to bind electrostatically to negatively charged LPS molecules of the outer membrane of bacteria [40], then enter the cytoplasm and inhibit multiple intracellular targets $[40,41]$. Although the exact intracellular targets for bactenecin action have not been identified, we found that TRK1 potassium transporter-deficient $C$. albicans cells had reduced susceptibility to bactenecin compared with wildtype cells, indicating that ion transporters may be possible cytoplasmic-localized effectors involved in the killing mechanism of fungi [27].

Human neutrophil defensins

Mammalian defensins are a family of cationic peptides that are divided into $\alpha$ - and $\beta$-defensin subfamilies on the basis of the connectivity of highly conserved cysteine residues. Human neutrophil defensins (HNP-1 to HNP-4) are stored in azurophilic granules of the neutrophil. They are transferred to phagolysosomes upon phagocytosis to kill ingested microbes. However, these peptides can also be released into the extracellular space, where they not only contribute to extracellular killing of microorganisms but also can recruit neutrophils, memory $\mathrm{T}$ cells, and immature dendritic cells to the site of infection. Human $\beta$-defensins (HBD-1 through HBD-4), expressed predominantly in epithelial cells of mucous membranes and in keratinocytes, are well studied in terms of active concentrations and spectrum of antimicrobial activity, but very little is known about their mechanism of action.

The precise mechanism for antibacterial activity of human neutrophil defensins has not yet been clearly defined, but it has been widely believed that defensins permeabilize membranes through multimeric pore formation [42]. It has been suggested that the cationic characteristic and amphipathic nature of antimicrobial peptides allows binding to and direct interaction with the lipid bilayer of cell membrane, leading to leakage of the internal aqueous content of the cell. Defensins contain $\beta$-sheet structures, a feature that enables formation of ion channels in model lipid membranes [43]. The presumed mechanism for their action includes (1) binding to anionic groups located outside of the bacterial membrane, (2) insertion into the membrane to form aggregates, and (3) finally produce an assembly of six or more dimers to form a pore as it was shown with Staphylococcus aureus [44]. Studies of $\alpha$ defensins did not reveal specificity toward a distinct target membrane, since they were active against bacteria, enveloped viruses, and fungi. However, defensins are able to discriminate between bacterial membranes and membranes of higher eukaryotic organisms by preferential interaction with anionic phospholipids that are predominant in prokaryotic cells [44].

The antifungal mechanism of action of defensins differs greatly from the nonspecific membrane permeabilization found against bacteria. HNP-1 induced a dose-dependent efflux of ATP from C. albicans cells that was inhibited by the same pharmacological agents (CCCP, DNP, and azide) and ionic conditions $\left(\mathrm{Ca}^{2+}\right.$ and $\left.\mathrm{Mg}^{2+}\right)$ found to inhibit Hst 5 killing of $C$. albicans [45]. HNP-1-induced efflux of cellular ATP occurred while cells were respiring and had polarized membranes, showing that membrane disruption was not the cause of selective loss of nucleotide [45]. Similarly, incubation of $C$. albicans cells with lethal doses of HBD-2 or HBD-3 did not result in loss of membrane integrity [46].

\section{Cathelicidin peptides and LL37}

LL37 belongs to the cathelicidins family, a group of proteins that are characterized by a highly conserved cathelin pro-sequence linked to a variable antimicrobial domain [47]. In C. albicans, the localization of LL37 was confined mainly to the cell perimeter in a pattern very similar to cells treated with Calcofluor White M2R (a specific cell wall marker), suggesting an association with 
the cell wall and/or cell membrane [47]. Prolonged treatment of these cells with the peptide caused a complete disappearance of the integrity of cellular membranes, resulting in leakage of ATP and other small nucleotides, but without loss of proteins larger than $40 \mathrm{kDa}$ [47].

The ability of LL37 to cause membrane perforation of the membrane in Actinobacillus actinomycetemcomitans [48] and other gram-negative bacteria has been shown to be dependent on more negatively charged cell membranes, compared with strains having lower zeta potential values [48]. Disintegration of the outer and inner membrane of $A$. actinomycetemcomitans resulted in cell membrane perforation, suggesting that the target of LL37 is the bacterial membrane and LPS. In this regard, it has been reported that Treponema denticola showed resistance to antimicrobial peptides due to the lack of LPS [48]. These results suggested that the strains with a highly negative charge are more susceptible to antimicrobial peptides among the same bacterial species as shown for A. actinomycetemcomitans and S. aureus [48, 49]. Interestingly, LL37 itself may be able to stimulate immune cells, since application of LL37 to LPS-primed monocytes induced a transient release of ATP and interleukin (IL)-1 beta release via the P2X(7) receptor without cell cytotoxicity [50] (Table 1).

\section{Temporins, dermaseptins, and nisin}

Other small cationic peptides that act upon pathogenic bacteria and fungi show similar patterns of membrane permeabilization and loss of intracellular ATP. However, this group of peptides appears to have more significant species-dependant membrane effects that include loss of larger intracellular molecules in addition to ATP.

Temporins are a family of linear 10-13 residue cationic peptides isolated from the skin of the European red frog Rana temporaria that are active against gram-positive bacteria and C. albicans. The reported mechanism of antimicrobial action against Leishmania includes rapid induction membrane potential collapse as well as lowering the intracellular ATP levels [51]. Temporins induce permeation of the membrane (characterized by alteration of the $\mathrm{K}^{+}$and $\mathrm{Na}^{+}$gradient across the membrane and influx of

Table 1 Effects of cationic peptides on fungal cells

\begin{tabular}{llll}
\hline & $\begin{array}{l}\text { ATP } \\
\text { release }\end{array}$ & $\begin{array}{l}\text { Membrane } \\
\text { lysis }\end{array}$ & $\begin{array}{l}\text { PI uptake/ } \\
\text { ion transport }\end{array}$ \\
\hline Histatin 5 & Yes & No & Yes \\
Lactoferricin & Yes & No & Yes \\
Bactenecin & ND & ND & Yes \\
HNP-1 & Yes & ND & Yes \\
Cathelicidin & Yes & Yes & ND \\
\hline
\end{tabular}

vital dyes such as the SYTOX Green) simultaneously with inhibition of parasite proliferation [51].

Dermaseptin S3 has been shown to exert antimicrobial action by specific interactions of the amphipathic $\alpha$-helix moiety with the plasma membrane phospholipids, leading to cell permeabilization [52]. Studies on Saccharomyces cerevisiae have shown that exposure to the peptide results in significant loss of ADP and ATP and other UV-absorbing compounds into the surrounding medium and that this efflux was significantly increased at, or near, a neutral $\mathrm{pH}$. Upon exposure to dermaseptin, the majority of cells were rapidly permeabilized [53].

Nisin acts against Listeria monocytogenes in a combination of effects due to membrane disruption, such as depolarization and inhibition of the respiratory chain and the loss of critical cellular components such as ATP and intracellular $\mathrm{K}^{+}$ions to the external environment [54].

Role of ATPe on host immune response and microbial clearance Since a prominent feature of nearly all cationic peptides upon interaction with target microbes is the loss of ATP into the extracellular media, it has been proposed that such localized increases in ATP levels results in potent immunomodulatory activities, such as chemoattraction of human monocytes, $\mathrm{T}$ cells and mast cells, and induction of chemokines $[55,56]$. Thus, peptide-induced ATP release from microbial pathogens not only serves to disable targeted cells, but may also be an important signal for the host immune response. The ability of innate defense peptides, chiefly defensins and cathelicidins, to contribute to stimulation of immune responses has prompted them to be reclassified as "alarmins" in recognition of their immunomodulatory role [57]. It is possible that peptide-induced loss of ATP at the site of infection may serve as an additional signal to activate the innate immune system $[58,59]$. Thus, antimicrobial peptides may possess biological functions extending beyond the innate immune response, perhaps synergizing with ATPe in stimulating immune response.

Conclusions One of the unifying signatures following interactions of cationic peptide agents with target microbial cells is the rapid release of intracellular nucleotides, especially ATP, into the surrounding environment. A longheld paradigm for the microbicidal action of cationic peptides is that killing of target microbial cell membranes occurs chiefly as a result of creation of membrane pores in the organism, leading to leakage of ions and metabolites. While it is likely that these effects contribute to ultimate microbial cell death, a mounting body of evidence supports additional or complementary mechanisms, so that membrane permeabilization alone appears insufficient to cause cell death. Rather, depletion of intracellular ATP and loss of ion balance are likely to have metabolic effects that cause 
cell cycle arrest rather than immediate cell death. A localized increase in EATP at the site of contact of cationic peptides with infecting microorganisms may itself be a significant signal for host immune enhancement to promote eradication of pathogenic microbes.

\section{References}

1. Brown KL, Hancock RE (2006) Cationic host defense (antimicrobial) peptides. Curr Opin Immunol 18:24-30

2. Lin AL, Johnson DA, Patterson TF et al (2001) Salivary anticandidal activity and saliva composition in an HIV-infected cohort. Oral Microbiol Immunol 16:270-278

3. McPhee JB, Hancock RE (2005) Function and therapeutic potential of host defence peptides. J Pept Sci 11:677-687

4. Koczulla AR, Bals R (2003) Antimicrobial peptides: current status and therapeutic potential. Drugs 63:389-406

5. Yeaman MR, Yount NY (2003) Mechanisms of antimicrobial peptide action and resistance. Pharmacol Rev 55:27-55

6. Lehrer RI, Ganz T (1996) Endogenous vertebrate antibiotics. Defensins, protegrins and other cysteine-rich antimicrobial peptides. Ann N Y Acad Sci 797:228-239

7. Kagan BL, Ganz T, Lehrer RI (1994) Defensins: a family of antimicrobial and cytotoxic peptides. Toxicology 87:131-149

8. Matsuzaki K (1998) Magainins as paradigm for the mode of action of pore forming polypeptides. Biochim Biophys Acta 1376:391-400

9. Koo SP, Bayer AS, Yeaman MR (2001) Diversity in antistaphylococcal mechanisms among membrane-targeting antimicrobial peptides. Infect Immun 69:4916-4922

10. Lehrer RI, Barton A, Daher KA et al (1989) Interaction of human defensins with Escherichia coli. Mechanism of bactericidal activity. J Clin Invest 84:553-561

11. Chapple DS, Hussain R, Joannou CL et al (2004) Structure and association of human lactoferrin peptides with Escherichia coli lipopolysaccharide. Antimicrob Agents Chemother 48:2190-2198

12. Blumenthal KM, Seibert AL (2003) Voltage-gated sodium channel toxins: poisons, probes, and future promise. Cell Biochem Biophys 38:215-238

13. Williams K (1997) Interactions of polyamines with ion channels. Biochem J 325:289-297

14. Jouirou B, Mouhat S, Andreotti N et al (2004) Toxin determinants required for interaction with voltage-gated $\mathrm{K}+$ channels. Toxicon 43:909-914

15. Mathie A, Wooltorton JR, Watkins CS (1998) Voltage-activated potassium channels in mammalian neurons and their block by novel pharmacological agents. Gen Pharmacol 30:13-24

16. Edgerton M, Koshlukova SE, Lo TE et al (1998) Candidacidal activity of salivary histatins. Identification of a histatin 5-binding protein on Candida albicans. J Biol Chem 273:20438-20447

17. Li XS, Reddy MS, Baev D et al (2003) Candida albicans Ssa1/2p is the cell envelope binding protein for human salivary histatin 5 . J Biol Chem 278:28553-28561

18. Ruissen AL, Groenink J, Helmerhorst EJ et al (2001) Effects of histatin 5 and derived peptides on Candida albicans. Biochem $\mathrm{J}$ 356:361-368

19. Veerman EC, Nazmi K, Van't Hof W et al (2004) Reactive oxygen species play no role in the candidacidal activity of the salivary antimicrobial peptide histatin 5. Biochem J 381:447-452

20. Murakami Y, Shizukuishi S, Tsunemitsu A et al (1991) Binding of a histidine-rich peptide to Porphyromonas gingivalis. FEMS Microbiol Lett 66:253-256
21. Koshlukova SE, Araujo MW, Baev D et al (1999) Salivary histatin 5 induces non-lytic release of ATP from Candida albicans leading to cell death. J Biol Chem 274:18872-18879

22. Xu Y, Ambudkar I, Yamagashi H et al (1999) Histatin 3-mediated killing of Candida albicans: effect of extracellular salt concentration on binding and internalization. Antimicrob Agents Chemother 43:2256-2262

23. Baev D, Li X, Edgerton M (2001) Genetically engineered human salivary histatin genes are functional in Candida albicans: development of a new system for studying histatin candidacidal activity. Microbiology 147:3323-3334

24. Baev D, Li XS, Dong J et al (2002) Human salivary histatin 5 causes disordered volume regulation and cell cycle arrest in Candida albicans. Infect Immun 71:3251-3260

25. Baev D, Rivetta A, Li XS et al (2003) Killing of Candida albicans by human salivary histatin 5 is modulated, but not determined, by the potassium transporter TOK1. Infect Immun 71:3251-3260

26. Baev D, Rivetta A, Vylkova S et al (2004) The TRK1 potassium transporter is the critical effector target for killing of Candida albicans by the cationic protein, Histatin 5. J Biol Chem 279:55060-55072

27. Vylkova S, Li XS, Berner JC et al (2006) Distinct antifungal mechanisms: beta-defensins require Candida albicans Ssal protein, while Trk1p mediates activity of cysteine-free cationic peptides. Antimicrob Agents Chemother 50:324-331

28. Di Virgilio F (1995) The P2Z purinoreceptors: an intriguing role in immunity, inflammation and cell death. Immunol Today 16:524-528

29. Ferrari D, Pizzirani C, Adinolfi E et al (2004) The antibiotic polymyxin B modulates $\mathrm{P} 2 \mathrm{X} 7$ receptor function. J Immunol 173:4652-4660

30. Lopes-Buesa P, Pfund C, Craig EA (1998) The biochemical properties of the ATPase activity of a $70-\mathrm{kDa}$ heat shock protein (Hsp70) are governed by the C-terminal domains. Proc Natl Acad Sci U S A 95:15253-15258

31. Gyurko C, Lendenmann U, Troxler RF et al (2000) Candida albicans mutants deficient in respiration are resistant to the small cationic salivary antimicrobial peptide histatin 5. Antimicrob Agents Chemother 44:348-354

32. Lupetti A, Paulusma-Annema A, Welling MM et al (2000) Candidacidal activities of human lactoferrin peptides derived from the $\mathrm{N}$ terminus. Antimicrob Agents Chemother 44:3257-3263

33. Viejo-Diaz M, Andres MT, Fierro JF (2004) Effects of human lactoferrin on the cytoplasmic membrane of Candida albicans cells related with its candidacidal activity. FEMS Immunol Med Microbiol 42:181-185

34. Viejo-Diaz M, Andres MT, Fierro JF (2004) Modulation of in vitro fungicidal activity of human lactoferrin against Candida albicans by extracellular cation concentration and target cell metabolic activity. Antimicrob Agents Chemother 48:1242-1248

35. Ulvatne $\mathrm{H}$, Haukland $\mathrm{HH}$, Olsvik O et al (2001) Lactoferricin B causes depolarization of the cytoplasmic membrane of Escherichia coli ATCC 25922 and fusion of negatively charged liposomes. FEBS Lett 492:62-65

36. van der Kraan MIA, van Marle J, Nazmi K et al (2005) Ultrastructural effects of antimicrobial peptides from bovine lactoferrin on the membranes of Candida albicans and Escherichia coli. Peptides 26:1537-1542

37. Lupetti A, Paulusma-Annemma A, Senesi S et al (2002) Internal thiols and reactive oxygen species in candidacidal activity exerted by an N-terminal peptide of human lactoferrin. Antimicrob Agents Chemother 46:1634-1639

38. Tanida T, Okamoto T, Ueta E et al (2006) Antimicrobial peptides enhance the candidacidal activity of antifungal drugs by promot- 
ing the efflux of ATP from Candida cells. J Antimicrob Chemother 57:94-103

39. Monk BC, Niimi K, Lin S et al (2005) Surface-active fungicidal D-peptide inhibitors of the plasma membrane proton pump that blocks azole resistance. Antimicrob Agents Chemother 49:57-70

40. Skerlavaj B, Romeo D, Gennaro R (1990) Rapid membrane permeabilization and inhibition of vital functions of gram-negative bacteria by bactenecins. Infect Immun 58:3724-3730

41. Sadler K, Eom KD, Yang JL et al (2002) Translocating prolinerich peptides from the antimicrobial peptide bactenecin 7 . Biochemistry 41:14150-14157

42. Sahl HG, Pag U, Bonness $\mathrm{S}$ et al (2005) Mammalian defensins: structures and mechanism of antibiotic activity. J Leukoc Biol $77: 466-475$

43. Kagan BL, Ganz T, Lehrer RI (1994) Defensins: a family of antimicrobial and cytotoxic peptides. Toxicology 87:131-149

44. Chen H, Xu Z, Peng L et al (2005) Recent advances in the research and development of human defensins. Peptides 27:931940

45. Edgerton M, Koshlukova SE, Araujo MW et al (2000) Salivary histatin 5 and human neutrophil defensin 1 kill Candida albicans via shared pathways. Antimicrob Agents Chemother 44:3310 3316

46. Vylkova S, Nayyar N, Li W et al (2007) Human beta-defensins kill Candida albicans in an energy-dependent and salt-sensitive manner without causing membrane disruption. Antimicrob Agents Chemother 51:154-161

47. den Hertog AL, van Marle J, van Veen HA et al (2005) Candidacidal effects of two antimicrobial peptides: histatin 5 causes small membrane defects, but LL37 causes massive disruption of the cell membrane. Biochem J 388:689-695

48. Ouhara K, Komatsuzawa H, Yamada S et al (2005) Susceptibilities of periodontopathogenic and cariogenic bacteria to antibacterial peptides, \{beta\}-defensins and LL37, produced by human epithelial cells. J Antimicrob Chemother 55:888-896
49. Peschel A, Otto M, Jack RW et al (1999) Inactivation of the dlt operon in Staphylococcus aureus confers sensitivity to defensins, protegrins, and other antimicrobial peptides. J Biol Chem 274:8405-8410

50. Elssner A, Duncan M, Gavrilin M et al (2004) A novel P2X7 receptor activator, the human cathelicidin-derived peptide LL37, induces IL-1 beta processing and release. J Immunol 172:4987-4994

51. Mangoni ML, Saugar JM, Dellisanti M et al (2005) Temporins, small antimicrobial peptides with leishmanicidal activity. J Biol Chem 280:984-990

52. Jouenne T, Mor A, Bonato $\mathrm{H}$ et al (1998) Antibacterial activity of synthetic dermaseptins against growing and non-growing Escherichia coli cultures. J Antimicrob Chemother 42:87-90

53. Soote PJ, Holyoak CD, Bracey D et al (1998) Inhibitory action of a truncated derivative of the amphibian skin peptide dermaseptin s3 on Saccharomyces cerevisiae. Antimicrob Agents Chemother 42:2160-2170

54. Abee T, Krockel L, Hill C (1995) Bacteriocins: modes of action and potentials in food preservation and control of food poisoning. Int J Food Microbiol 28:169-185

55. Bowdish DME, Davidson DJ, Scott MG et al (2005) Immunomodulatory activities of small host defense peptides. Antimicrob Agents Chemother 49:1727-1732

56. Selsted ME, Ouellette AJ (2005) Mammalian defensins in the antimicrobial immune response. Nat Immunol 6:551-557

57. Oppenheim JJ, Yang D (2005) Alarmins: chemotactic activators of immune responses. Curr Opin Immunol 17:359-365

58. Hanley PJ, Musset B, Renigunta V et al (2004) Extracellular ATP induces oscillations of intracellular $\mathrm{Ca}^{2+}$ and membrane potential and promotes transcription of IL-6 in macrophages. Proc Natl Acad Sci U S A 101:9479-9484

59. Granstein RD, Ding W, Huang J et al (2005) Augmentation of cutaneous immune responses by ATP gamma $\mathrm{S}$ : purinergic agonists define a novel class of immunologic adjuvants. J Immunol 174:7725-7731 